\title{
Star Forming Galaxies and AGN Hosts: The Seagull Wings
}

\author{
Grażyna Stasińska ${ }^{1}$, Roberto Cid Fernandes ${ }^{2}$, Abílio Mateus ${ }^{3}$, Laerte \\ Sodré Jr. ${ }^{3}$, and Natalia V. Asari ${ }^{2}$ \\ ${ }^{1}$ LUTH, Observatoire de Meudon, 92195 Meudon Cedex, France \\ ${ }^{2}$ Departamento de Física, Universidade Federal de Santa Catarina, Florianópolis, SC, Brazil \\ ${ }^{3}$ Departamento de Astronomia, IAG-USP, Rua do Matão 1226, 05508-090, São Paulo, Brazil
}

\begin{abstract}
Using photoionization models applied to the data from the Sloan Digital Sky Survey (SDSS) we propose a physically motivated dividing line in the $[\mathrm{OIII}] / \mathrm{H} \beta$ vs $[\mathrm{NII}] / \mathrm{H} \alpha(\mathrm{BPT})$ diagram between normal star forming (NSF) galaxies and AGN hosts. We also propose a new diagnostic diagram which can be used for optical spectra of galaxies with redshifts up to $z=1.3$.
\end{abstract}

Keywords. galaxies: active — galaxies: starburst — emission lines: surveys

\section{Introduction}

In the BPT diagram, NSF galaxies and AGN hosts from the SDSS form two sequences, which look like the wings of a seagull. The Kewley et al. (2001) and Kauffman et al. (2003) lines to distinguish both classes are too "generous" for NSF galaxies. We propose a better dividing line, given by: $y=\left(-30.787+1.1358 x+0.27297 x^{2}\right) \tanh (5.7409 x)-31.093$, where $y=\log [\mathrm{OIII}] / \mathrm{H} \beta$, and $x=\log [\mathrm{NII}] / \mathrm{H} \alpha$.

We then propose a new diagnostic diagram, where NSF galaxies and AGN hosts are divided by the line: $D_{n}(4000)=-0.15(\log x+1)+1.7$, where $x=\max (\mathrm{EW}[\mathrm{OII}]$, $\mathrm{EW}[\mathrm{NeIII}])$.

See Stasińska et al. (2006) for more details.
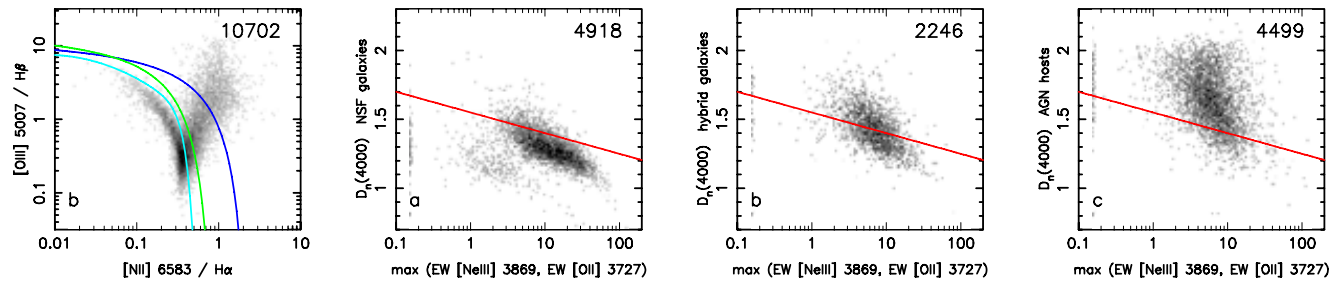

Figure 1. a: The BPT diagram for 10702 galaxies from the SDSS DR2 (Abazadjian et al. 2002). The Kewley line, the Kauffmann line and our line are shown from top to bottom. b, c, d: $D_{n}(4000)$ versus max (EW[OII], EW[NeIII]) for NSF galaxies, hybrid galaxies and AGN galaxies respectively. The red line is the adopted boundary between NSF and AGN galaxies.

\section{Acknowledgements}

We thank the SDSS team, and acknowledge financial support from CNPq and FAPESP.

\section{References}

Abazajian, K., et al., 2004, AJ, 128, 502

Kauffmann, G. et al., 2003, MNRAS, 346, 1055

Kewley, L. J. et al., 2001, ApJ, 556, 121

Stasińska, G., Cid Fernandes, R., Mateus, A., et al., 2006, MNRAS, 371, 972 